\title{
Les filles sortent (de) leurs bulles : Pénélope Bagieu, Margaux Motin, Eva Rollin, Diglee... Un nouveau genre de BD féminine.
}

\section{Séverine Olivier \\ Université Libre de Bruxelles}

Dans la première décennie des années 2000, Pénélope Bagieu, Margaux Motin, Anne Guillard, Aude Picault, Eva Rollin, Diglee, Nathalie Jomard... envahissent les rayons des librairies avec une nouvelle forme de bande dessinée féminine. Pour ces auteures qui sont loin d'être de parfaites inconnues, parfois grâce à leur blog, et dans un contexte éditorial favorable caractérisé par la multiplication des productions et la volonté des éditeurs de séduire un public féminin, le succès commercial est alors au rendez-vous. En 2011, trois ans après sa sortie, Ma vie est tout à fait fascinante de Pénélope Bagieu s'était déjà vendu à 75000 exemplaires (Gindensperger) alors que La Tectonique des plaques de Margaux Motin (2013) aurait franchi en 2015 le cap des 100000 exemplaires (Cosseron).

Même si les bédéistes citées n'atteignent pas toutes ces chiffres de vente, elles participent bien à un nouveau phénomène éditorial. La percée de cette BD féminine, étiquetée «BD girly », s'explique par son contenu et par la création d'une héroïne du quotidien à l'image de ses auteures et de ses lecteurs (ou plus certainement lectrices). Mais n'est-elle pas due également au caractère hybride de ces nouvelles vignettes et nouveaux phylactères? Il ne s'agit pas d'évoquer ici l'hybridité de la bande dessinée alliant images et textes, mais bien de questionner son hybridité définie comme intermédialité. Intermédiatique, telle est en effet cette $\mathrm{BD}$ du féminin, née dans et grâce à d'autres médias qui ont contribué à la caractériser. Elle doit sa genèse à l'Internet et plus spécifiquement à la blogosphère, ainsi qu'à la presse féminine avec laquelle elle entretient des liens étroits et, enfin, à la chick lit apparue avec Le Journal de Bridget Jones, dans le sillage duquel elle s'inscrit.

Ainsi précisée, cette intermédialité ne confine-t-elle pas les bédéistes « girly » aux marges de la bande dessinée en les conduisant à créer une BD populaire ${ }^{1}$ et féminine? Dans quelle mesure en effet le contexte d'émergence de cette production et les stratégies qu'il suppose ne les ont pas menées, voire contraintes, à composer (oserait-on dire "fabriquer »?) une bande dessinée de grande consommation, mais également une BD de genre, au sens de catégorie littéraire certes, mais aussi et surtout au sens sexué du terme? À moins que, dans ce contexte profitable, ces stratégies de soumission aux

${ }^{1}$ Dans tous les sens du terme. 
industries culturelles ne soient assumées et conscientes? Peut-être sont-elles en réalité, aussi, pour ces auteures une façon de s'assurer une première publication, et partant, d'ouvrir la voie à des projets plus personnels et reconnus sur l'échelle des hiérarchies culturelles ${ }^{2}$ ? C'est donc le caractère intermédiatique de la « BD girly» que cet article se propose de mettre en lumière, pour mieux interroger ensuite cette intermédialité : cloisonne-t-elle ou non cette production et ses créatrices dans le sous-champ de la culture populaire féminine?

\section{BD d'un quotidien « girly "}

Qu'il s'agisse d'autobiographies, d'autofictions ou parfois de fictions, les BD de Pénélope Bagieu et consorts mettent toutes en scène des personnages féminins tournés vers eux-mêmes et leur existence pas « tout à fait fascinante » et plutôt ordinaire. Anti-héroïnes ou héroïnes du quotidien (Lallemand 20), ces figures féminines se confient et dévoilent leurs obsessions de jeunes femmes au commencement de leur vie professionnelle, en couple ou en quête de l'homme idéal, partagées entre raison et passion démesurée pour les «fringues » et chaussures de marque, biberonnées à la culture de masse et influencées par les canons de mode et de beauté édictés par les médias. Un détour par la quatrième de couverture d'Autobiographie d'une fille $G A G A$ de Diglee permet de mieux saisir leurs caractéristiques :

Je m'appelle Diglee, j'ai 22 ans, une sœur dingo et géniale, un homme roux, une Best friend forever qui me supporte depuis 10 ans, une mère super canon, et trop de cellulite pour mon IMC, malgré mes chorées endiablées sur Lady Gaga. Et comme ma vie intéresse tout le monde, hein, baah... j'en ai fait un blog. Et puis de ce blog, j'en ai fait un livre. Voilà. (Diglee)

La légèreté, la frivolité, voir l'inanité du propos (dont les auteures ne sont d'ailleurs pas dupes : Diglee le laisse entendre ci-dessus), ses clichés et poncifs liés aux femmes à la limite de la caricature et sa bonne dose de nombrilisme n'auraient pas contribué au succès de cette bande dessinée sans l'ironie qui la caractérise et l'autodérision dont les personnages et, par leur voix, leurs conceptrices font preuve. Alors que l'illustration de la banalité du quotidien favorise l'illusion référentielle et l'identification, l'humour qui en souligne la futilité assure la prise de distance et le défoulement. Réaliste, mais cathartique, car humoristique, telle est la bande dessinée étiquetée " girly ».

Cette dénomination polémique, sur laquelle je reviendrai et dont cherchent à se distancier, entre autres, Pénélope Bagieu et Diglee, doit sa

\footnotetext{
2 Même si d'aucuns voudraient voir s'effacer la dichotomie légitime/illégitime (Ducas citant notamment Eric Maigret), force est de constater qu'à l'heure où la BD est devenue un objet d'étude scientifique comme un autre, sa reconnaissance et sa consécration restent partielles et passent par le rejet, au sein du champ qui lui est propre, d'un ensemble de productions. Comme le rappelle Tanguy Habrand, la BD contemporaine n'est pas une, mais elle est deux, tiraillée entre l'édition indépendante et les industries culturelles. (Habrand).
} 
création à la presse et aux éditeurs. Terme anglais évoquant à la fois la féminité et le glamour, l'adjectif "girly» ${ }^{3}$ est devenu un argument de marketing et une étiquette efficace assurant la visibilité de productions qui se ressemblent par leur thématique et leur ton.

\section{BD intermédiatique : blog, presse et chick lit}

Associée aux blogs, à la presse féminine et à la chick lit, la BD girly, $\mathrm{BD}$ intermédiatique, doit ses origines et ses particularités à ces trois médias.

\section{a. Moi, je... de la toile à la planche}

Point de départ pour nombre d'auteures de BD girly : l'Internet. Pénélope Bagieu explique la genèse de son blog «Ma vie est tout à fait fascinante » (2007) par le besoin de souffler entre deux boulots d'illustration pour la publicité (Gindensperger). Diglee, en introduction d'Autobiographie d'une fille $G A G A$, revient sur les origines informatiques de sa $\mathrm{BD}$ et ajoute par ailleurs sur son blog, dans une perspective tout à fait identique à celle de Pénélope Bagieu :

J'ai ouvert mon blog en 2007, en parallèle du diplôme et de l'école en général, pour construire un petit «cocon» d'humour et de légèreté, un endroit qui me ressemblait et m'épanouissait plus que les boulots que nous demandait l'école (trop sombres et académiques pour moi). (Diglee, $b \log$ )

Quant à Margaux Motin, elle se dessine pianotant sur un clavier à la troisième planche de J'aurais adoré être ethnologue intitulée «Deux ou trois choses qu'il faut savoir sur moi ».

Lieu d'apparition de nouvelles expériences en littérature et en littérature populaire ${ }^{4}$, le Web 2.0 permet à chacun, dès la première décennie des années 2000, de s'improviser écrivain ou dessinateur et d'accéder à la reconnaissance avant même d'être édité sur le papier. Partagées, invitant aux commentaires, les productions de la toile, par leur interactivité, s'inscrivent dans un rapport nouveau au public récepteur. Le vecteur production/réception se transforme et se complexifie. "Prosommateur $»^{5}$ plus que consommateur, le récepteur produit lui-même pour partie ce qu'il consomme ou, du moins, en influence le contenu. En réagissant, commentant les productions qui lui sont proposées sur le Net, il convie leur

\footnotetext{
${ }^{3}$ Apparu dans la première décennie des années 2000, cet adjectif semble être employé dès 2009 2010 pour désigner les bandes dessinées évoquées ici avant de se populariser en 2011, ce que montrent une rapide recherche sur l'Internet et les articles de presse mis en ligne sur le sujet.

${ }^{4}$ Comme les blooks ou blouquins, livres publiés sur le net ou encore les fanfictions, récits inspirés par la culture médiatique (de best-sellers comme Harry Potter ou de films) et écrits par des amateurs qui désirent prolonger les aventures des personnages qu'ils ont lues ou vues.

${ }^{5}$ Terme utilisé notamment par Eva Illouz dans son analyse de Cinquante nuances de Grey de E. L. James, ce roman ayant pour origine l'écriture d'une fanfiction (Illouz 36-37)
} 
créateur à les modifier, les réorienter ${ }^{6}$. Ce dernier peut alors répondre aux attentes des internautes qui, en retour, par leurs clics, leur nombre de vues et leurs commentaires assurent sa publicité et son succès. Il sera peut-être ainsi plus facilement repéré par des éditeurs, dont la prise de risque se réduit considérablement, et dont l'image du métier semble évoluer. En effet, ils ne sont plus seulement des découvreurs de talents, mais aussi des «vigies du Net», contraints (et cela semble surtout vrai pour la littérature populaire) à garder un œil sur la toile pour être les premiers à se saisir d'un bu₹z littéraire et récupérer celui ou celle qui deviendra peut-être leur poule aux œufs d'or ${ }^{7}$. Fait de petites maisons d'édition dans un premier temps, cet intérêt pour les productions virtuelles d'internautes s'étend et touche d'autres éditeurs, ces derniers cherchant sur le Web un moyen de renouveler ou d'étoffer leurs catalogues à peu de frais.

Dans la première décennie des années 2000, le choix d'un blog dessiné, s'il peut s'expliquer par un besoin de libertés et un désir d'expérimentations graphiques, notamment chez les auteurs déjà professionnels, apparaît aussi, lorsqu'il est conçu entre autres en marge d'une activité plus ou moins liée à la $\mathrm{BD}$, comme un moyen de s'intégrer dans une communauté de dessinateurs-blogueurs: Diglee renvoie sur son blog à Margaux Motin, dont elle raconte la rencontre dans Autobiographie d'une fille GAGA. Margaux Motin se représente d'ailleurs elle-même à la dernière planche de cet ouvrage. Cette communauté virtuelle assure une forme de parrainage. Mais le recours au Web 2.0 pour les bédéistes en herbe est surtout une façon de se faire connaître de lecteurs et remarquer des éditeurs. Leur succès auprès d'un public qui, à la base, n'est pas nécessairement amateur de bandes dessinées et dont l'intérêt se porte parfois plus sur le contenu que sur la forme ${ }^{8}$ étant gage de publicité gratuite et de chiffres de vente éveille la curiosité des maisons d'édition, parfois non spécialistes du $9^{\mathrm{e}}$ art. Ainsi Pénélope Bagieu a-t-elle été repérée par l'éditeur Jean-Claude

\footnotetext{
${ }^{6}$ Il serait d'ailleurs intéressant d'envisager une approche génétique de la BD dont il est ici question : blogue et publication en album proposent-ils des dessins identiques? Ou, au contraire, comme le laisse entendre Diglee en introduction de son premier ouvrage, les dessins sont-ils retravaillés de l'un à l'autre ? Et le sont-ils en fonction de commentaires reçus ? Comment, en outre, sont-ils sélectionnés par leurs auteures et qui en détient les droits au final ?

D'autre part, se pose la question de la réception de ces albums conçus à partir d'un blog : quel est donc le public qui s'aventure à acheter l'œuvre papier et pourquoi s'y aventure-t-il alors que les dessins sont disponibles, sous un autre format certes, sur le net ? Quant au mode de lecture, il semble différent du blogue au livre : le média virtuel propose au jour le jour une lecture antéchronologique sur le mode de la verticalité (déroulement de la page Internet) alors que la lecture de l'album se veut plus linéaire. ${ }^{7}$ Je pense notamment au succès récent d'E.L. James, auteure de Cinquante nuances de Grey, roman tiré d'une fanfiction publiée sur un forum consacré à Twilight, la saga « vampire» de Stephenie Meyer. Ou encore, à la série After d'Anna Todd, fanfiction éditée depuis janvier 2015 en France en format livre par Hugo Roman.

${ }^{8}$ Julien Baudry indique en effet que le rapport au blog BD est le rapport d'un lecteur à un auteur et non d'un lecteur à une œuvre: ce qui intéresse les Internautes avant tout, ce sont les anecdotes personnelles, autobiographiques ou autofictionnelles que le blog raconte. (Baudry, «les blogues»)
} 
Gawsewitch alors que Margaux Motin ou Diglee sont publiées par Marabout. Passerelle entre le monde de la création amateur et le monde professionnel de la $\mathrm{BD}$, les blogs constituent alors un tremplin vers l'édition papier. Permettant aux auteurs d'émerger, ils sont un moyen de se faire publier.

La toile néanmoins, lieu d'apparition, mais aussi vitrine des blogs $\mathrm{BD}$, n'ouvre pas à elle seule la voie vers la publication papier. Cette dernière tient aussi à leurs particularités : les blogs dessinés dont il est question ici sont, par leur nature même ${ }^{9}$ et leurs spécificités autobiographiques ou autofictionnelles, une forme de journaux ${ }^{10}$. Or, Anne Kerebel a montré que c'est dans l'objectif d'être publié que s'inscrivait paradoxalement aujourd'hui le genre du journal :

Le journal marque le triomphe de l'individu dans une dimension particulière, qui est celle de l'intime. Il accueille les impressions, les émotions, les confessions d'une subjectivité, et pourtant l'idée de public, d'un public, d'un destinataire imaginaire, fantasmé, ou réel, voire d'une publication, est constamment présente. (Kerebel 109)

Les nouvelles technologies de la communication combinées à la culture du moi, au centrage sur l'individu (Kaufmann 19) et au culte de l'authenticité (Baetens) ont poussé l'intimité à se dévoiler, voire s'exhiber, via la blogosphère où elle s'exprime parfois en format illustré. Il apparaît par ailleurs, comme le remarque Laurence Brogniez, que plus largement, au-delà même de la toile, "l'une des tendances de fond» de la bande dessinée contemporaine passe par le récit du moi (Brogniez). Mémoires, autobiographie, autofiction, journal et blog, tels en sont les sous-genres et les modalités ${ }^{11}$. Ils répondent à la fois à un désir de l'auteur, mais également au besoin d'un public poussé, par le développement des réseaux sociaux entre autres, au voyeurisme. Aux facteurs technologiques dus à l'explosion du Web 2.0 qui modifient le circuit éditorial, se mêlent dès lors des facteurs sociologiques. Ensemble, ils expliquent la percée et la nature de cette bande dessinée.

Se dessinent donc ici les premières trames du contexte d'émergence de la BD girly, qui en oriente le sujet, la structure et le ton, et en explique le succès. Dans l'espoir de se faire remarquer (même s'il n'est pas reconnu explicitement comme tel ${ }^{12}$ ), elle naît sur un blog qui, répondant pour partie

\footnotetext{
${ }^{9}$ Etymologiquement, le mot blogue, contraction de «Web $\log »$, désigne un journal sur le Web.

${ }^{10}$ Le journal intime dans lequel le blogueur propose ses anecdotes du quotidien est selon Julien Baudry la forme la plus courante que prennent les blogs BD. (Baudry, «Les blogues »)

${ }^{11}$ Jan Baetens regrettait en 2004 que ces formes autobiographiques soient plus souvent masculines que féminines.

${ }^{12}$ Dans plus d'une interview, Pénélope Bagieu souligne qu'elle ne s'attendait pas à un tel succès. La référence au hasard ou à la Providence peut toutefois relever d'une posture d'auteur.
} 
aux attentes ou aux réactions d'Internautes, est contraint, s'il veut maintenir l'intérêt et fidéliser, d'être actuel et sans cesse actualisée ${ }^{13}$, rapide et/ou ludique à lire (Rouquette). À ses origines, la BD girly se présente donc comme le journal ou le carnet de bord d'un quotidien ordinaire, féminin, à l'image de ses auteures, divisé en brèves saynètes représentées en une ou deux planches ou billets humoristiques, légers et cathartiques.

\section{b. Du papier glacé des magazines aux vignettes}

L'apparition de cette BD sur des blogs n'explique pas à elle seule son contenu et son retentissement. La BD girly entretient aussi des liens étroits avec les magazines féminins qui ont également contribué à la mettre en lumière. Illustratrices de profession, ses auteures ont souvent collaboré avec la presse féminine, avant même la création de leur blog ou la publication qui en a découlé. Dans le magazine Muteen destiné à des femmes âgées d'une vingtaine d'années, Margaux Motin rédigeait et illustrait un billet mensuel intitulé «J'ai testé » (le siège massant, le rubber band, le simulateur d'aube...). À la dernière page de la revue suisse Femina, se déclinait la vie de Joséphine, personnage créé par Pénélope Bagieu. Et même s'il ne s'agit pas à proprement parler de presse féminine, les aventures d'Eva dessinées dans Voici par Aude Picault y renvoyaient par leur thématique : jeune femme qui « se cherche désespérément », bientôt trentenaire, en quête de l'homme idéal, Eva avait tout d'une fashionista ${ }^{14}$. Plus généralement, Elle, Cosmopolitan, Marie Claire, Madame Figaro, Grazia font appel à ces dessinatrices pour l'une ou l'autre illustration dans un article ou en couverture. À cela s'ajoutent, dans les pages de ces mêmes magazines, des publicités dessinées par ces illustratrices pour Canderel, Kellog's, Dim, Bourjois, Yves Rocher...

Au contraire du Web 2.0 investi comme un hobby ${ }^{15}$, l'illustration dans la presse féminine se présente comme un emploi, un moyen de subsistance $^{16}$. Mais qu'ils soient lieu de plaisir et défouloir ou source de revenus, le blog et le magazine féminin éclairent tous deux le contexte d'émergence des auteures de BD girly. Pratiquées en parallèle puisqu'elles se présentent sur deux plans différents (un volet «amateur» et un volet "professionnel»), ces activités servent de rampes de lancement aux dessinatrices en leur garantissant un lieu d'expression et sans doute un

\footnotetext{
${ }^{13}$ Anne Kerebel parle de "publication intime "in time" » à propos des journaux intimes publiés en lignes (108).

${ }^{14}$ C'est aussi dans la presse people que Pénélope Bagieu a opéré en créant en 2008 pour le lancement du magazine Oops le personnage girly de Charlotte.

${ }^{15}$ Même si Margaux Motin a conçu son blog au départ comme un book professionnel, ce dernier a vite évolué en blog $\mathrm{BD}$.

${ }^{16}$ Certaines auteures de BD girly sont aussi illustratrices d'album pour enfants. La seule auteure de BD girly qui n'a pas fait du dessin sa profession à part entière est Myriam Rak alias Mimi Stinguette. Autodidacte dans l'univers dessiné, elle exerce le métier de juriste (Pasamonik, « Myriam Rak »).
} 
public : se joint peut-être aux internautes un lectorat heureux de retrouver en format $\mathrm{BD}$ les illustrations et dessins de ses magazines féminins.

En leur offrant la possibilité de faire leurs preuves, la presse féminine devient une forme de vitrine des auteures de BD girly qu'elle promeut par ailleurs. Ainsi, lorsque sort J'aurais adoré être ethnologue de Margaux Motin, Muteen qui s'était déjà fait l'écho de son blog (Locatelli, «Mon espace ») lui dédie un encart positif, le magazine rappelant d'ailleurs qu'elle y fut une chroniqueuse dévouée (Locatelli, «She's back »). De même, Femina consacre deux pages à Pénélope Bagieu lors de la publication du premier volume de Joséphine, un article presque schizophrénique. Lieu de création du personnage, le magazine interroge pourtant l'auteur sur ses origines :

FEMINA Comment est né le personnage de Joséphine?

PENELOPE BAGIEU Quand Femina m'a commandé cette BD, j’ai imaginé un personnage... (Schmidt 18)

La lecture de la revue de presse de cette dessinatrice reprise sur son blogue montre, quant à elle, que ses premiers pas furent soulignés, toute proportion gardée, plus par les magazines féminins que par d'autres médias de presse (presse généraliste, culturelle...). Puisqu'elle en a permis le déploiement, la presse féminine accorde un certain intérêt à cette nouvelle forme de bande dessinée. Et les auteures de lui renvoyer l'ascenseur : leurs personnages de fiction se plongent de temps à autre dans la lecture de Grazia ou Glamour.

Au contraire des héroïnes de chick lit qui manient l'ironie autour des prescriptions des magazines et dont le discours est à leur égard des plus ambivalents (Olivier, « De la presse » 84-86), les protagonistes de la BD girly semblent moins les dénoncer. Leur lecture se veut certes légère (lecture de cabinet ou de plage, test à faire avec une copine ${ }^{17}$ ), mais elle n'est pas nécessairement critique: les auteures se souviennent sans doute de ce qu'elles doivent à la presse féminine et leurs personnages peuvent difficilement lyncher ce qui contribue à leurs revenus ${ }^{18}$. Exception faite de Joséphine qui, à la première planche du tome 2, « lit trop la presse » et à qui les conseils ne conviennent pas et surtout de Valentine d'Anne Guillard. Toutefois, la critique n'est jamais acerbe : moqueuse, elle cherche plutôt à susciter le rire. Reprenant les codes de la couverture d'un magazine féminin, la BD V alentine la parodie (voir image 1). Chaque numéro renvoie à un titre bien connu qu'il détourne: «Elle décoloration », «Seule \& Jolie »,... Les intitulés de l'illustré fictif sont facétieux : «Vos poils, vos kilos, votre mec...

\footnotetext{
${ }^{17}$ Voir chez Margaux Motin « Mon père ce héros » et « J'auras fait un très mauvais Robinson Crusoé » (Motin, J'aurais adorê). Et chez Diglee, « Cruel dilemme » (Diglee).

${ }^{18}$ Margaux Motin indique dans ses remerciements : « À Cath’ et Mélu, les Muteen en chef, en souvenir du bon vieux temps, et parce que vous avez été les premières à me donner ma chance, tout est parti de vous, je n'oublie pas!» (Motin, J'aurais adorê).
} 
Comment vous en débarrasser avant l'été!... » ou encore « Métro Boulot... Gastro ». À l'intérieur, les vignettes alternent avec témoignages, interviews, horoscope, publicités et tests créés de toute pièce.

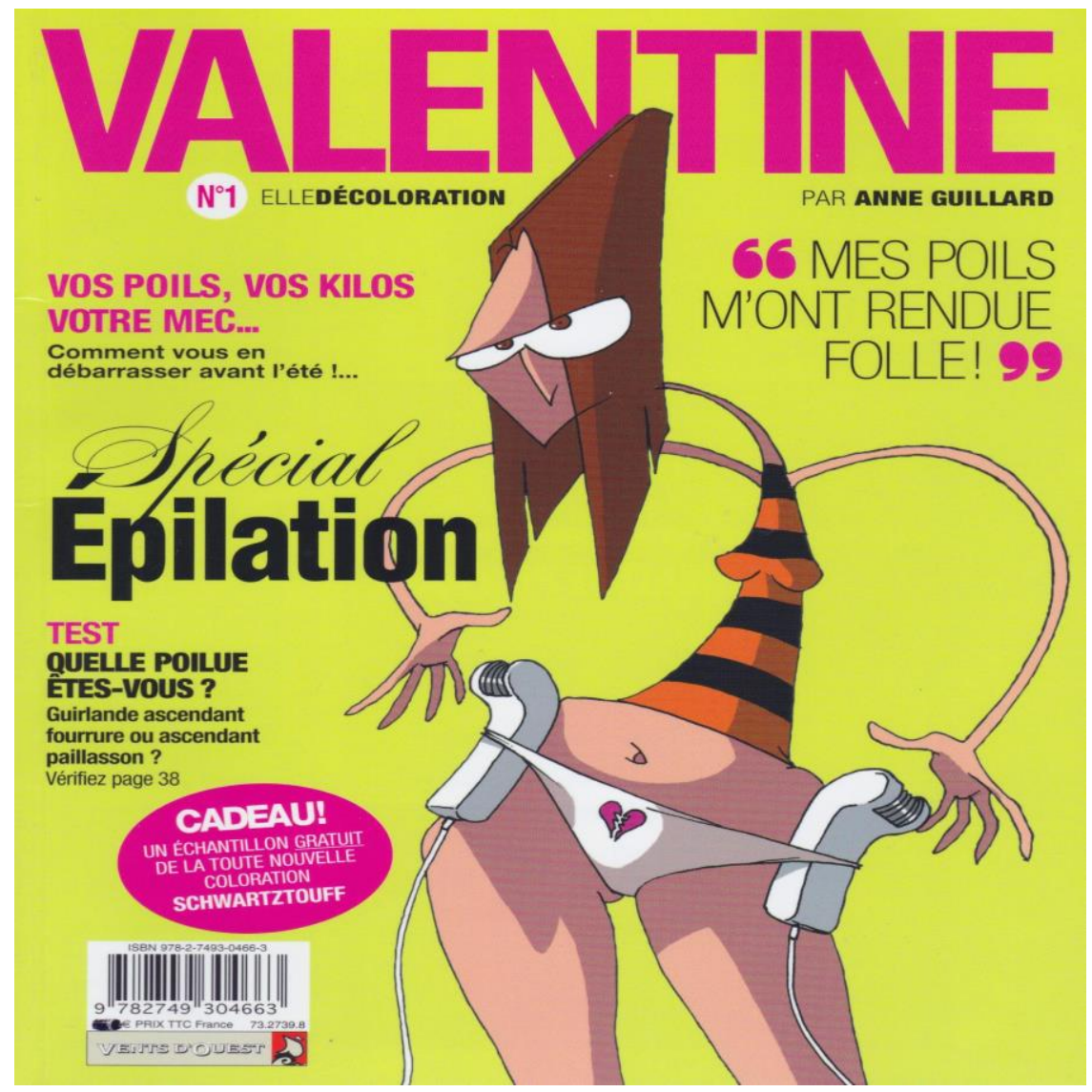

Image 1 : Crédits. Couverture (Guillard). Droits Réservés.

Qu'elle l'évoque en passant, qu'elle s'en inspire explicitement, qu'elle la raille ou qu'elle en détourne les codes, la BD girly entretient donc un rapport étroit avec la presse féminine : par le métier de ses auteures, mais aussi parce que ces médias s'inscrivent dans une culture dite de masse marquée par la société de consommation dont les magazines féminins, mais aussi les avatars de Pénélope Bagieu et compagnie sont les produits. Au-delà de leurs discours réciproques, ces médias auxquels s'associe la publicité publiée dans les magazines et illustrée par les bédéistes girly se rejoignent donc sur le plan thématique. Leur composition les rapproche également puisqu'elle fusionne contenu et placement de produits : à côté d'un article sur des questions de beauté se dévoile une publicité pour un cosmétique tandis que les créatrices de BD girly, illustratrices pour de grandes marques, glissent de temps à autre dans leur (auto) fiction le nom de l'une ou l'autre d'entre elles. Cette pratique du name dropping - voire, pour créer un néologisme, du "picture dropping» chez Margaux Motin insérant dans ses 
planches une photo de chaussures Louboutin - éclaire la passion des auteures de $\mathrm{BD}$ girly pour la mode et les dernières tendances, tendances dont elles sont parfois des préscriptrices ${ }^{19}$. Leur blogroll renvoie en effet aux sites de marques ou de créateurs et Graz̧ia de solliciter les conseils de Diglee en matière de tenues vestimentaires :

G : Un petit peu de mode avant de finir : vos personnages sont toujours très lookés. Quelles sont les tendances de cette saison que l'on retrouvera bientôt dans vos illustrations et sur votre blog?

$\mathrm{D}$ : Ma foi, je ne sais pas. J'ai lâché la paillette et le doré depuis fort longtemps. Mes lecteurs et moi-même sommes perdus! Je reste quand même attachée au pastel, quand il fait bien froid : pull bleu ciel, col contrasté, collants blancs en laine et manteau rose pâle. Ah si : je veux un manteau rose dans la lignée du Carven, fabuleusement marshmallow de cet hiver. (Volfson)

Plus largement, les femmes que ces médias dépeignent et/ou auxquelles ils s'adressent reposent sur des représentations féminines identiques, qualifiées de postféministes: girly ou glamour, les femmes figurées revendiquent leur féminité tout en clamant leur indépendance et leur libertée $^{20}$. Un qualificatif que Pénélope Bagieu résume en quelques mots pour le Journal du Dimanche: "Pour moi, il ne doit pas y avoir d'un côté les coquettes et de l'autre les intelligentes. Sans cela, le combat de nos mères n'aura servi à rien. » (Barret 5).

Les figures féminines des magazines, des publicités et des BD examinées dans cet article, reflets parfois de leurs auteures, ont donc de nombreux points communs qui tiennent autant à leurs préoccupations (shopping et bien-être) qu'à leurs représentations (" féministes féminines »). Alors que le blogue délimitait avant tout la forme (diaristique, brève, actualisée et humoristique) de cette nouvelle forme de bande dessinée, les liens qu'elle tisse avec la presse féminine déterminent son contenu.

\section{c. Sur la vague de la chick lit}

Tandis que les blogs et les magazines féminins dessinent les conditions favorables d'émergence de la $\mathrm{BD}$ girly, son rapport à la chick lit en dévoile plutôt le contexte. La percée de cette bande dessinée est immanquablement due au succès d'une nouvelle littérature populaire et féminine dont le Journal de Bridget Jones de l'anglaise Helen Fielding (1996) et Sex and the City de l'américaine Candace Bushnell (1996) restent les modèles.

Ces récits, publiés d'abord sous forme de chroniques respectivement dans The Independent et The New York Observer, et leurs multiples clones se centrent sur les aventures de citadines trentenaires en quête du Prince

\footnotetext{
${ }^{19}$ Pénélope Bagieu a d'ailleurs créé sa propre ligne de lingerie pour Etam.

${ }^{20}$ La problématique du postféminisme dans les médias est éclairée notamment par Rosalind Gill à laquelle je renvoie pour plus de détails sur le sujet.
} 
Charmant, de la dernière petite robe à la mode, d'un moyen de combler le négatif de leur compte bancaire et de supprimer leurs dépendances, en quête surtout d'un équilibre existentiel. Racontées sous la forme de confessions et prenant le plus souvent l'apparence d'un journal, ces aventures tracent le visage d'une nouvelle figure féminine antihérö̈que ou ordinaire, dont l'archétype est Bridget Jones ${ }^{21}$. Les copies de ce personnage à succès ${ }^{22}$ envahissent les rayons des librairies et les éditeurs s'emparent alors de l'étiquette chick lit, inventée dans le but de souligner la frivolité de cette littérature, pour la réinvestir positivement et en faire un outil de marketing. Dès les années 2000, la chick lit fleurit partout: les auteures connues du genre, Helen Fielding, Candace Bushnell, mais aussi Isabel Wolff, Sophie Kinsella, Lauren Weisberger, côtoient de nombreuses collections pensées par les maisons d'édition. En France, Red Dress Ink créée en 2005 est éditée par Harlequin aux côtés de Girls in the City de Marabout, Mille Comédies de Belfond ou Girls de Fleuve Noir.

Ces auteurs et ces collections, les auteures de la BD girly les connaissent bien puisque l'illustration de leurs jaquettes relève de leurs activités professionnelles d'illustratrices indépendantes. Les couvertures de la collection Girls in the City chez Marabout et celles chez J'ai lu des bestsellers d'Helen Fielding ou des récits d'Isabelle Alexis, auteure française de chick lit, ont pris forme sous les crayons de Margaux Motin. Chez Pocket, les romans de Lauren Weisberger se parent des dessins de Nathalie Jomard (Stiletto Blues à Hollywood, édition de 2011), alors que Diglee et Anne Guillard illustrent la teen chick lit ou chick lit pour adolescentes en croquant pour l'une Queen Betsy, personnage de MaryJanice Davidson, et pour l'autre Allie, figure créée par Meg Cabot.

C'est donc dans cet environnement éditorial familier que Pénélope Bagieu et ses consœurs conçoivent de nouvelles Bridget Jones : alors que «Pénélope vit $[\ldots]$ en compagnie de son chat rose, de sa collection de chaussures et de ses tracas quotidiens : sa réticence à faire du sport, sa mère envahissante... », Joséphine « travaille dans un bureau avec plein de gens qui ne connaissent pas son nom, mais remarquent ses achats compulsifs » et « il ne lui manque pas grand-chose, à part peut-être l'homme idéal, pour pouvoir faire des courses pour deux, imaginer des soirées torrides et partir en weekend romantique. $»^{23}$ "Célibattante » et toujours prête à prendre de bonnes résolutions comme l'héroïne d'Helen Fielding, indépendante, mais sexy juchée sur ses talons aiguilles comme Carrie Bradshaw de Candace Bushnell,

\footnotetext{
${ }^{21}$ Pour plus de détails sur la chick lit, voir mon article sur le sujet (Olivier, « La chick lit»).

${ }^{22}$ Publié sous forme de feuilleton en 1995, le Journal de Bridget Jones sort en format livre en 1996 en anglais et en 1998 en français avant d'être adapté au grand écran en 2001.

${ }^{23}$ Quatrièmes de couverture de Ma vie est tout a fait fascinante (Le Livre de Poche, 2014) et de Joséphine (Le Livre de Poche, 2010).
} 
ou shopaholic, immature, endettée et renonçant à l'assumer comme Becky Bloomwood de Sophie Kinsella, l'héroïne de la BD girly ressemble à s'y méprendre à l'hérö̈ne de chick lit. Comme elle, elle a d'ailleurs évolué, devenant mère (Joséphine : Change de camp; Mademoiselle: L'arme fotale; Le Petit grumeau illustré) ou affrontant le cap difficile de la quarantaine (40 ans! What the fuck!! d'Emmanuelle Teyras ou Scènes de mon âge d'Agnès Abécassis, par ailleurs auteure de romans chick lit) : autant de déclinaisons rappelant les sous-genres de la chick lit. En effet, cette dernière prend parfois la forme de la mom lit ou de la hen lit et conte respectivement les déboires d'une jeune maman ou celles d'une femme non plus dans la trentaine, mais âgée d'une quarantaine ou cinquantaine d'années. Et si, dans la BD girly, le dessin, plus que les mots, rend compte des aventures des figures féminines, elles sont comme dans les romans contées avec humour sur le ton de la connivence. La pré-publication sur un blog favorise d'ailleurs la forme de la confession et le clin d'œil au lecteur, également caractéristiques de la chick. lit : il n'est pas rare que la lectrice se fasse interpeller par le personnage féminin qui se confie. Le blogue rappelle en outre la genèse des best-sellers pionniers du genre, chroniques journalistiques publiées périodiquement au départ, et évoque les Nouvelles Technologies de l'Information et de la Communication (mails, SMS, etc.) dont sont friandes les héroïnes de chick lit. Comparable à cette publication féminine sur le plan thématique, la $\mathrm{BD}$ girly l'est donc également et, à plus d'un titre, sur le plan formel. Cette BD que le Web 2.0 et la presse féminine ont concouru à faire émerger tout en traçant ses spécificités de forme et de fond s'est par conséquent, en raison de ces spécificités justement, fait l'écho de la chick lit qui, grâce à sa couverture médiatique, lui a garanti son envol et son succès. Plus qu'un écho, la BD girly représente peut-être ainsi le visage qu'a pris en France la chick lit, production anglo-américaine d'origine.

S'il est vrai que la naissance de cette littérature populaire s'inscrit dans un contexte sociologique international (marqué par la montée du célibat) expliquant son succès et son développement au-delà du champ culturel anglo-américain, force est de constater que les romans francophones étiquetés chick lit n'ont pas connu le retentissement de leurs homologues anglophones ou celui de la BD girly francophone. Plusieurs explications éclairent ce phénomène. Les auteures - le plus souvent des journalistes à l'instar de leurs homologues d'outre-Manche et d'outre-Atlantique - restent plutôt rares ${ }^{24}$. En France, l'intériorisation des hiérarchies culturelles n'incite pas les écrivains aspirant à la reconnaissance littéraire à s'adonner à la littérature de grande consommation. En outre, les romancières francophones marquées auteures de chick lit rejettent cette étiquette vu ses

\footnotetext{
${ }^{24}$ Pensons à Sylvie Overnoy, rédactrice en chef de Cosmopolitan et auteure entre autres de SOS Amour, ou Sophie Fontanel, journaliste chez Elle et auteure de Fonelle est amoureuse, qui tient par ailleurs un blogue signé Fonelle (http://www.dailyelle.fr/etre-fonelle).
} 
connotations péjoratives : grand public et creuse, telle serait cette production que la presse francophone s'est par ailleurs empressée de critiquer. La traduction des termes chick lit en « littérature de poulettes » en est l'exemple le plus frappant. Ce sont donc principalement des romans traduits qui occupent les étals des librairies. Il n'est par conséquent pas interdit de penser que la BD girly a pris la place laissée vacante par la chick lit francophone. $\mathrm{Ou}$ pour le dire autrement : la chick lit francophone a pris la forme de la BD girly.

Certes, la BD girly n'est pas uniquement française: Marabout a publié en 2015 Journal d'une emmerdeuse de la dessinatrice argentine vivant à Barcelone, Augustina Guerrero. Quant à Eva Rollin, elle est d'origine québécoise. Cependant, le phénomène reste principalement hexagonal. Or, comme l'indique Julien Baudry, les blogues BD dont est issue la BD girly sont une «exception française » qui tient sans doute à une «coïncidence chronologique entre un engouement général des auteurs et des lecteurs pour l'autobiographie dessinée et un mouvement de publication en ligne sur un outil adapté à cette dernière. » (Baudry, « La bande dessinée ») La chick lit se caractérisant par sa forme diaristique n'a-t-elle donc pas trouvé sur la toile française le meilleur moyen d'expression : le blogue $\mathrm{BD}$ ne serait-il pas un support plus adéquat qu'un livre - car moins légitime - pour cette littérature légère, d'autant que la bande dessinée elle-même reste encore pour une large part déconsidérée? À ses origines, la BD girly publiée sur le Web semble donc avoir constitué la parfaite synthèse entre un courant autobiographique dessiné en ligne et le courant de la chick lit. Son succès une fois assuré sur la toile, restait aux éditeurs à profiter de l'engouement suscité par cette bande dessinée en ligne et à la transformer en un produit marketing qui, associé à Bridget Jones et à son journal, fut vite étiqueté et décliné en sous-genres, comme l'ont été les ouvrages d'Helen Fielding et de ses émules.

En conclusion, confluent entre le blog et la BD, entre la presse féminine et la $\mathrm{BD}$, entre la chick lit et la $\mathrm{BD}$, mais aussi entre le blog $\mathrm{BD}$ et la chick lit, la $\mathrm{BD}$ girly est à la croisée de différents médias à l'origine de son développement, de son déploiement et de son succès, mais aussi de ses particularités et plus largement de sa place dans le champ de la bande dessinée.

\section{BD de genre, auteures en marge?}

\section{a. Une BD d'un nouveau genre?}




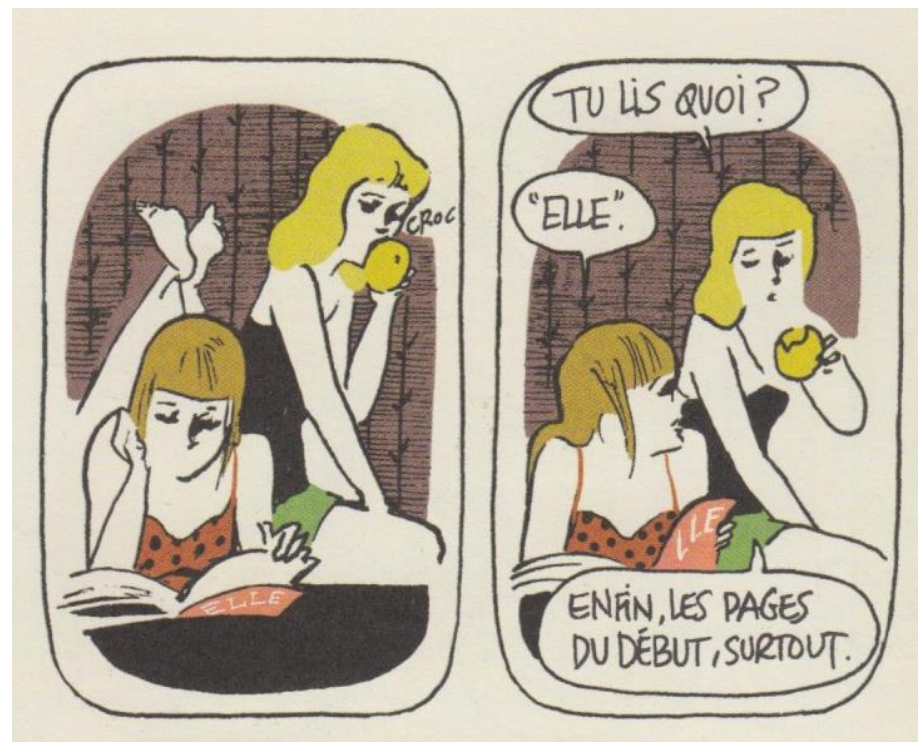

Image 2 : Crédits : (Antico 24). Droits Réservés.

Si la BD girly est un genre, elle l'est moins par l'existence de règles à suivre pour s'y conformer que par le regroupement empirique d'albums renvoyant à un contexte social, virtuel, littéraire et éditorial particulier, mais différent, du moins stylistiquement parlant. De là se dégage parfois une impression diffuse d'un fourre-tout réunissant Joséphine de Pénélope Bagieu et Girls don't cry de Nine Antico, illustratrice chez Muteen dont Les inRocks indiquent d'ailleurs qu'elle «dynamite» par «son ton acidulé et son trait vintage » la BD de filles (Norot) (voir image 2). S'y retrouvent également Eva Rollin et Nathalie Jomard qui s'amusent toutes deux des affres de la maternité, mais dont l'esthétique est radicalement dissemblable (voir images 3 et 4). Ces BD partagent pourtant une série de points communs déjà détaillés ci-dessus. Déterminés par les médias qui les ont vus naitre et les ont mises en lumière (blogues, magazines féminins et romans chick lit), ils sont à l'origine de leur classification en genre, classification que renforce la création de l'étiquette de $\mathrm{BD}$ girly. Sous cette bannière presque générique, bien que commerciale avant tout, est présenté un ensemble de productions populaires, divertissantes - d'aucuns diraient frivoles -, et féminines. 


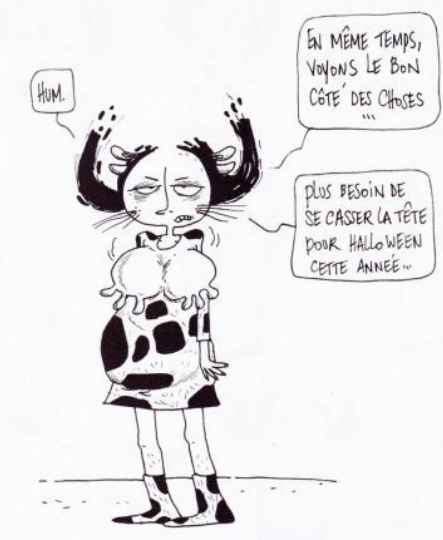

Image 3 : Crédits : (Rollin 40). Droits Réservés.

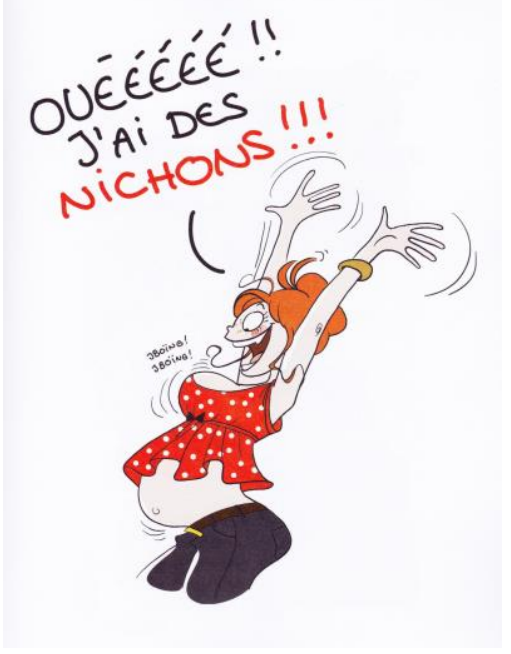

Image 4 : Crédits : (Jomard). Droits Réservés.

Par la forme autofictionnelle de leurs récits, l'intime étant souvent associé à la féminité, par les sujets traités (le quotidien et les relations amoureuses), par leurs personnages, par leur métier d'illustratrices et par leur sexe, Pénélope Bagieu, Margaux Motin, Diglee... auraient créé une BD de femmes ou du moins une BD présentée comme telle. Sa catégorisation genrée n'est pas uniquement due à ses caractéristiques et à celles de ses créatrices, elle dépend aussi de son contexte et de son support d'émergence qui ont parfois conduit ses auteures à concevoir des figures féminines à la limite de la caricature. Leurs marges de manœuvre comme illustratrices professionnelles, tracées par un directeur artistique et un cahier des charges pour un client précis, étaient déjà plutôt limitées. Mais Pénélope Bagieu parle 
aussi d'« exercice de style » pour sa BD Joséphine (Florent). Et, Aude Picault d'indiquer à propos d'Eva, commande de Voici: «Eva ce n'est pas moi, c'est un gros cliché!» (Lallemand 23). Les stratégies éditoriales ont donc contribué à enfermer et clicher cette bande dessinée et ses auteures dans un carcan féminin.

\section{b. Des filles en marge...}

L'étiquette BD girly, inventée ou reprise par les éditeurs, éclaire des volontés marchandes évidentes, une dérive que dénonce d'ailleurs Pénélope Bagieu : «Les éditeurs s'acharnent en effet à publier n'importe quelle nana grâce à laquelle ils pourront vendre une couverture rose, même si elle n'a pas de talent.» (Pudlowski), Mais envisagée comme une marque ou presque, cette étiquette a aussi et surtout contribué à cloisonner les auteures qu'elle désigne dans un univers BD bien particulier :

Quand l'étiquette du « Girly » est arrivée, j’ai cru bon d'en jouer, de me l'approprier, d'en rire. Un élément isolé mis en lumière par-ci par-là pour la blague, c'était drôle. Jusqu'à ce que cet infime, cette bribe, cet échantillon prenne le dessus, englobe tout mon travail, et annihile tout effort d'écriture, de réflexion, d'émotion.

Sur mon blog, j'ai parlé de tout. J'ai parlé de la famille, du rapport au corps, de la prise d'indépendance, d'amitié, de harcèlement de rue, de règles, de voyages, de découvertes, de deuil, de chats, des [sic] bouffe, d'amour, de sexe, parfois... de relations, beaucoup. En 7 ans de confessions, je réalise que n'en ressort souvent que ce qu'on veut faire passer pour le plus creux, le plus inutile. En voulant rire de ma superficialité ponctuellement, je l'ai fait exister davantage, je l'ai érigée en vérité, et me suis enfermée dans une image réductrice que je pensais pourtant combattre.

(Diglee, blog)

Pour exister, les bédéistes sujets de cet article ont dû, comme le souligne Diglee, « jouer du girly », s'y conformer, se soumettre aux stratégies des industries culturelles et adopter parfois une posture de femme futile. Tout en s'attaquant à la perversité de cette BD stéréotypée et à son caractère dangereux pour les lectrices, critique séculaire adressée à toute production féminine populaire, la blogueuse et dessinatrice Tanxxx dénonce et interroge : «Et quoi ? nous autres, femmes dessinatrices, on est condamnées à être publiées dans des trucs de gonzesse débile, à causer de mascara, dans un ghetto bien loin des vraies éditions qu'on propose par ailleurs ? $\gg^{25}$ Cette interrogation et cette dénonciation, reprises dans un «auto-droit» de réponse à son attaque formulée contre la $\mathrm{BD}$ girly ${ }^{26}$, rejoignent les propos

\footnotetext{
${ }^{25}$ Attaque de la blogueuse Tanxxx retirée du net, suite au ramdam médiatique qu'elle a suscité (les articles de presse sur le sujet sont légion), mais relayée par Chantal Montellier et Florie Boy sur le blogue de Chantal Montellier (http://www.montellier.org/spip.php?article207). Tanxxx est revenue sur ses propos, dans un auto-droit de réponse daté du 8 juillet 2015. Elle les nuance les considérant $a$ posteriori comme le reflet d'une misogynie intégrée (http://tanxxx.free-h.fr/bloug/).

${ }^{26}$ "Ce qui ne va pas, [...], c'est que tu n'as pas compris que les blogues "girly" ne le sont que parce que des éditeurs (ou plus largement la société) ont voulu les désigner comme tels, que parler de
} 
des auteures qu'elle avait fustigées dans cette même attaque. Ainsi, Pénélope Bagieu explique :

Moi quand j'ai voulu dessiner des superhéros, on m'a dit 'super, il faudrait que ce soit des superhéroïnes, et qu'elles utilisent leur pouvoir pour faire les soldes'. Le message de ces éditeurs-là, c'est 'tu es une femme donc tu t'intéresses aux fringues et aux chats mignons'» (Pudlowski)

Se conformant aux attentes des éditeurs et à celles supposées du lectorat, les auteures de $\mathrm{BD}$ girly ont pris le risque de s'enfermer dans une image clichée, réductrice, qu'évoque ci-dessus l'auteure d'Autobiographie d'une fille GAGA. Aussi, et vu leurs conditions d'émergence, leurs bandes dessinées sont avant tout présentées comme des BD de filles, faites par des filles, pour des filles exclusivement. De ce fait, elles sont rejetées par la critique.

En 2004, Jan Baetens soulignait qu'une bande dessinée autobiographique était jugée excellente si elle racontait l'histoire d'un antihéros. Pourtant, alors que la BD girly dessine le parcours d'une figure féminine autofictionnelle au nombre de défauts incalculables, rares sont les critiques qui l'encensent. Au contraire, avant même sa parution, la voilà déjà condamnée, parfois pour des motifs contradictoires. Myriam Rak, alias Mimi Stinguette, s'étonne :

En ce qui concerne mes éditeurs, je souhaite avant toute chose souligner l'amusante contradiction dans les reproches qui leur sont faits : dans les mêmes commentaires, à quelques lignes d'écart, d'un côté, on accuse La Boîte à bulles d'être des opportunistes avides d'argent et de ventes faciles, et d'un autre, on qualifie mon livre (que personne n'a ni lu ni vu, je le rappelle) d'invendable et à peine regardable. (Pasamonik, « Myriam Rak »)

Inféodées à l'économie de marché, les productions de Pénélope Bagieu, Diglee, Margaux Motin ou Myriam Rak se retrouvent soumises, aliénées à leur identité genrée ${ }^{27}$ et immédiatement disqualifiées car féminines. Or, se défendant de faire du féminin, ces créatrices indiquent qu'elles s'intéressent plutôt à la peinture du quotidien, un quotidien croqué en une planche dont Sempé est le "modèle absolu» (Lemaire). Une façon de répondre aux critiques qui dénoncent leur superficialité (un défaut souvent associé aux femmes) et de légitimer leur création?

macarons ou de shopping n'est typiquement féminin que dans la tête de misogynes. (...) Tout n'est pas si simple que décréter que telle publication est merdique, c'est oublier que les femmes sont victimes du rôle qu'on leur assigne, et les fustiger parce qu'elles s'y conforment (et nier par la même occasion que ça puisse être autre chose que s'y conformer) fait totalement partie du modèle patriarcal. » (http://tanxxx.free-h.fr/bloug/).

${ }^{27}$ Pourtant, il ne faut pas nécessairement avoir un vagin pour apprécier ses livres, clame Diglee (Diglee, blog). Les commentaires postés sur les blogs des dessinatrices émanent aussi de lecteurs masculins. Et que faire de Pacco, blogueur dont la $\mathrm{BD}$ Maé est associée à l'univers de la $\mathrm{BD}$ girly puisqu'elle raconte avec humour et dans une perspective identique au Petit Grumeau illustré de Nathalie Jomard le quotidien d'un parent (père ici) et de sa fille de deux ans? 


\section{c. Sortir des cases}

Les stratégies éditoriales qui ont conduit à projeter la BD girly dans un sous-champ de la bande dessinée populaire et féminin n'y ont pas pour autant cloîtré définitivement ses dessinatrices. La promotion qu'elles leur ont faite et le succès qu'elles ont connu ont permis à ces dernières d'ouvrir des portes fermées jusque-là. Diglee évoque ainsi son dernier projet, l'histoire d'une femme dans les années 20 intitulée Anna, en indiquant que, refusé par le passé par les maisons d'édition, il était aujourd'hui prêt d'aboutir: «Il fallait juste du temps. Du temps pour que les éditeurs me fassent confiance sur d'autres terrains, tout en profitant de l'ampleur grandissante du phénomène « girly » avant qu'il ne s'essouffle. » (Diglee, blog)

En déclenchant et en profitant du «phénomène girly », les auteures sont sorties de leur blogue et de leur activité d'illustratrices. Elles ont publié leurs premiers albums BD qui ont parfois été primés et la notoriété gagnée alors, bien qu'elle semble les avoir conduites aux marges de la bande dessinée, à un enfermement dans une production grand public et sexuée, leur a paradoxalement offert l'opportunité de faire leurs preuves : la garantie d'un nom synonyme de rentabilité leur a donné l'occasion de s'envoler vers d'autres horizons.

Leurs premières publications reprenant la sélection d'illustrations humoristiques publiées sous forme de carnet de bord chez Jean-Claude Gawsewitch ou Marabout ont attiré l'œil d'éditeurs BD tels que Delcourt et ont ouvert la voie à des bandes dessinées plus conséquentes, plus (auto)fictionnelles qu'autobiographiques, où les croquis presque spontanés ${ }^{28}$ du quotidien ont été remplacés par des planches reliées par un fil conducteur. De $M a$ vie est tout à fait fascinante à Joséphine ( $c f$. ci-dessus images 2 et 3), d'Autobiographie d'une fille GAGA à Forever Bitch, de Mademoiselle à Les citadines $^{29}$, de J'aurais adoré être ethnologue à La tectonique des plaques, les thèmes n'ont pas changé, mais les BD sont davantage scénarisées ${ }^{30}$. Margaux Motin indique à propos de La tectonique des plaques:

Les précédents étaient plus bruts, parce que ça fonctionnait sur le système de la note de blog. Il y avait moins de travail de structure parce que c'était une petite intention à chaque fois. Là, on est sorti de la note de blog. Je me suis posé des vraies questions scénaristiques. (Lemaire)

\footnotetext{
${ }^{28}$ Cette spontanéité tient au blog, même si avant d'être publiés en album, les dessins sont parfois revus et corrigés.

${ }^{29}$ Les citadines dessinées par Eva Rollin qui content la vie de quatre trentenaires à la recherche de l'homme idéal sont scénarisées par Adeline Blondieau.

${ }^{30}$ La revue Newième art souligne quant à elle l'évolution de Karine Bernadou. S'appuyant sur le genre de la BD girly qu'elle a pratiqué, son travail se veut toutefois, selon Clément Lemoine, plus original dans Canopée : «c'est le même trait, le même thème, la même féminité revendiquée dès la couverture, avec un sein à découvert. Mais une féminité inquiète, aux prises avec un décor oppressant, bien loin de la futilité moyenne du quotidien. » (Lemoine).
} 
Petit à petit toutefois sont apparus de nouveaux sujets : après Moi je, les carnets de dessins d'Aude Picault, toujours introspectifs, abordent le suicide de son père. Et même si Papa publié par l'Association en 2006 à l'instigation de Jean-Christophe Menu laisse ensuite place à Eva, JF se cherche désespérément, Aude Picault lassée de «ces portraits irréels de femmes qui règlent leurs problèmes en s'achetant des chaussures » (Reber) est partie en mer pour en revenir avec Transat, le récit de son voyage. Pénélope Bagieu a, quant à elle, scénarisé et dessiné Cadavre exquis édité chez Gallimard (collection Bayou), son premier « vrai » récit pour reprendre les critiques de presse, sous l'impulsion de Joann Sfar avec lequel elle a ensuite créé Stars of the Stars. Et, avec Boulet au scénario, elle a signé La Page Blanche chez Delcourt en 2012. De la critique du monde de l'édition à la recherche de soi, Pénélope Joli Cœur qui continue à croquer son existence sur son blog met ses crayons au service d'autres thématiques. Ces dernières lui ont d'ailleurs valu un certain succès critique. Pour Le Monde, Pénélope Bagieu, décorée par la suite Chevalier des Arts et des Lettres et exposant ses œuvres au Festival d'Angoulême, confirmait avec Cadavre exquis « son brio avec un premier "vrai récit" prouvant sa capacité « de dépasser la "littérature pour poulettes" [...] option BD et de camper de vrais personnages. » (Labé) ${ }^{31}$ Rien de «vrai» alors dans les «historiettes » de Pénélope Joli Cœur pourtant largement autobiographiques? Pas assez de psychologie, de profondeur pour le récit humoristique de ses aventures du quotidien sans doute, mais peut-être aussi impossibilité de reconnaittre une production divertissante et féminine aux chiffres de vente importants... ${ }^{32}$ Enfin, outre le thème, c'est le style de leurs $\mathrm{BD}$ qui a évolué, tel celui de Diglee qui, de ses « égéries » en cartes postales à Anna, modifie les contours de ses figures féminines (voir son blog).

Désormais publiées par des éditeurs du $9^{\mathrm{e}}$ art, les auteures de BD girly, ont pris d'autres chemins au fil de leurs rencontres... des rencontres bien souvent masculines. Se pose dès lors une question : ont-elles donc besoin des hommes pour sortir de leurs bulles féminines? Pour Aude Picault : Jean-Christophe Menu et Boulet avec qui, entre autres, elle a signé le blog de Chicou-Chicou. Joann Sfar, Guy Delcourt, Boulet, mais également les rédacteurs de la revue Papier, Lewis Trondheim et Yannick Lejeune, pour Pénélope Bagieu. Illustrant la couverture du second numéro de cette revue aux styles les plus divers, elle y signe aussi une histoire courte. Pour Margaux Motin : Pacco, son associé au crayon ${ }^{33}$ comme dans la vie. Obion pour

\footnotetext{
${ }^{31}$ Cadavre exquis a reçu le prix SNCF à Angoulême et le prix DBD du meilleur album humour.

${ }^{32}$ Sur le rapport du discours critique à la création féminine en BD, voir l'article de Jean-Louis Tilleuil. Il relève une certaine dyssimétrie entre les compte rendus des productions $\mathrm{BD}$, dyssimétrie hiérarchique valorisant le masculin (Tilleuil). Une comparaison systématique de ce que dit la presse des blogs $\mathrm{BD}$ et des carnets de notes masculins et féminins pourrait se révéler intéressante.

${ }^{33}$ Voir : Very Bad Twinz signé à quatre mains par Pacco et Margaux Motin chez Fluide Glacial (2011).
} 
Gally : connue pour son «blogue d'une grosse » à l'origine de Mon Gras et moi rattaché à la $\mathrm{BD}$ girly, elle signe avec son blogueur de compagnon Love blogue chez Delcourt. Et que dire d'Hélène Bruller, créatrice de Je veux le prince charmant et ex-femme de Zep avec qui elle a plus d'une fois collaboré ? Émergeant seules avec leur blog et/ou leur ouvrage girly dans le champ de la BD, ces auteures semblent cependant y avoir gravi les échelons grâce au sexe fort. Si elles ont poussé sans aide les portes du succès, restait peut-être aux hommes, dans un univers où le masculin l'emporte encore ${ }^{34}$, à les maintenir ouvertes.

\section{En guise de conclusion...}

Ce ne sont pas des pionnières et elles ne sont pas les seules. Mais par leurs productions, Pénélope Bagieu, Margaux Motin, Diglee, Nathalie Jomard et d'autres encore, si elles n'ont pas concouru à asseoir le féminin dans le $9^{\mathrm{e}}$ art, l'ont contraint à s'y intéresser, à se préoccuper de la création, mais aussi de la réception de la BD par les femmes. C'est sans doute d'ailleurs ce lectorat en expansion qu'ont tenté de toucher, sans succès toutefois ${ }^{35}$, Fluide Glamour, devenu Fluide G., ou à sa suite, le magazine Bisou. Hors série de Fluide Glacial, décalé, féminin, sexy, caricatural et à l'humour incisif, Fluide $G$. illustré par des auteures comme Margaux Motin ou Diglee, rappelait par ses couvertures et l'intitulé de ses numéros ("Spécial Grosse et Moche », par exemple) les Valentine d'Anne Guillard (voir image 5). Si ce n'est que, comme pour Bisou de l'éditeur BD Delcourt, c'est la BD qui avait cette fois investi le féminin (Pasamonik) sans doute parce que le féminin a investi la BD.

\footnotetext{
${ }^{34}$ La polémique née au début de l'année 2016 autour du Grand Prix d'Angoulême et de la liste des nominés en est une parfaite illustration : la sélection opérée par les directeurs du Festival, accusés de sexisme, ne reprenait le nom d'aucune femme (Courval).

${ }^{35}$ Ces périodiques ne sont plus publiés.
} 


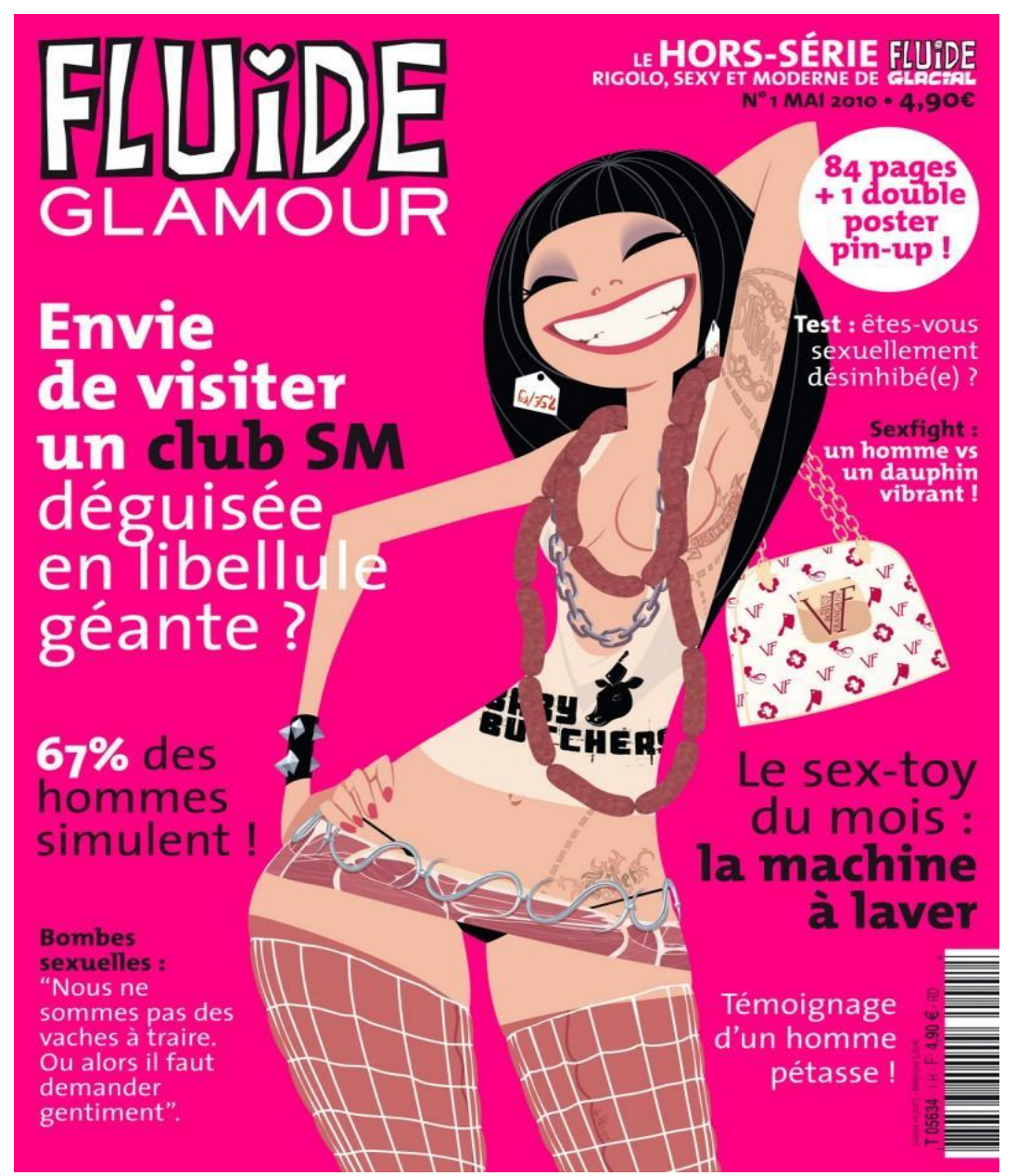

Image 5 : Crédits : Fluide Glamour. Le Hors-Série Fluide. Rigolo, sexy et moderne de Glacial. Mai 2010, $\mathrm{n}^{\circ}$ 1. Droits Réservés

Avec les auteures de BD girly, s'est démultiplié le nombre d'héroïnes féminines figurées dans la bande dessinée. Certes, d'aucuns les jugent stéréotypées et superficielles. Toutefois, sous l'impulsion de ces personnages et de leurs créatrices, la bande dessinée a continué d'intégrer des talents féminins. En outre, en renforçant ce mouvement initié avant elles ${ }^{36}$, les auteures de BD girly ont appâté un public nouveau, un public de lectrices, avec lequel il faut désormais compter. Parce qu'elle est un véritable phénomène, un phénomène intermédiatique lié autant à la chick lit et à la presse féminine qu'aux blogues (à la différence près que ces derniers ont permis à ses auteures d'être repérées au-delà de la sphère féminine), la BD girly a popularisé ou banalisé l'idée que la bande dessinée peut être au sujet des femmes, par les femmes et pour les femmes. En somme, le $9^{\mathrm{e}}$ art peut aussi parler des femmes, être aussi conçu par des femmes et plaire aussi à des

\footnotetext{
${ }^{36}$ Voir entre autres pour quelques chiffres l'article de Jean-Louis Tilleuil (120-121).
} 
femmes. Sans que cette triple potentialité ne conduise nécessairement à une bulle sexuée exclusive de créations féminines pensées par des auteures féminines à destination d'un public féminin sur des sujets prétendument féminins. 


\section{Bibliographie}

Antico, Nine. Girls don't cry. Paris : Glénat, 2010.

Baetens, Jan. «Autobiographies et bandes dessinées. Problèmes, enjeux, exemples. » Belphégor 4.1 (2004). Web. 13 juillet 2015.

Bagieu, Pénélope. Ma vie est tout à fait fascinante. Paris : Jean-Claude Gawsewitch, 2008.

Bagieu, Pénélope. Joséphine. Paris : Jean-Claude Gawsewitch, 2008.

Bagieu, Pénélope. Joséphine. 2. Même pas mal. Paris : Jean-Claude Gawsewitch, 2009.

Bagieu, Pénélope. Joséphine. 3. Change de camp. Paris: Jean-Claude Gawsewitch, 2010.

Bagieu, Pénélope. Cadavre exquis. Paris : Folio, 2011.

Bagieu, Pénélope et Boulet. La Page blanche. Paris : Delcourt, 2012.

Bagieu, Pénelope. Pénélope Bagieu. Ma vie est tout à fait fascinante. Web. 6 juillet 2015.

Barret, Anne-Laure. «Pénélope Bagieu : L’idole des filles. » Le Journal du Dimanche. 17 octobre $2010: 5$.

Baudry, Julien. «Histoire de la bande dessinée numérique française. Partie 2: la bande dessinée numérique à l'assaut du web (1996-2004).» Neuvième art 2.0 (2012). Web. 13 juin 2015.

Baudry, Julien. «Histoire de la bande dessinée numérique française. Partie 3 : les blogues bd, une spécificité française ?». Newvième art 2.0 (2012). Web. 13 juin 2015.

Brogniez, Laurence. «Féminin singulier : les desseins du moi. Julie Doucet, Dominique Goblet. » Textyles 36-37 (2010). 1 juin 2013. Web. 2 juin 2015.

Cosseron, Sophie. « La BD pour filles, le phénomène qui cartonne. » Femme actuelle. 16 janvier 2015. Web. 29 mai 2015.

Courval, Sophie. "Angoulême: une polémique révélatrice du machisme ordinaire dans le milieu de la bande dessinée. ».Les inRocks. 7 janvier 2016. Web. 20 janvier 2016.

Diglee. Autobiographie d'une fille Gaga. Paris : Marabout, 2011.

Diglee. Diglee. Web. 6 juillet 2015.

Ducas, Sylvie. «La littérature aujourd'hui: populaire?» Bulletin des bibliothèques de France 1 (2014). Web. 14 juillet 2015.

Fielding, Helen. Le Journal de Bridget Jones. Traduit de l'anglais par Arlette Stroumza. Paris : J'ai lu, 2001.

Florent, Fabrice. « Pénelope Bagieu. L’interview. Ne l'appelez plus Pénélope Jolicoeur sinon elle mord». wnw.madmoiZelle.com. septembre 2008. Web. 10 juillet 2015.

Gill, Rosalind. Gender and the Media. Cambridge : Polity Press, 2007.

Gindensperger, Sophie. «Dans la case fille. » Libération. 2 juillet 2011. Web. 29 mai 2015.

Guillard, Anne. Valentine. N.1:Elle Décoloration. Paris : Vents d'Ouest, 2005. 
Guillard, Anne. magtulipe.com. Web. 6 juillet 2015.

Habrand, Tanguy. "La "récupération" dans la bande dessinée contemporaine. » Textyles 36-37 (2010). 1 j.uin 2013.Web. 14 juin 2015.

Illouz, Eva. Hard Romance: Cinquante nuances de Grey et nous. Traduit de l'anglais et de l'allemand par Frédéric Joly. Paris : Seuil, 2014.

Jomard, Nathalie. Le petit grumeau illustré. Paris : Michel Lafon, 2009.

Jomard, Nathalie. Petit précis de grumeantique. Web. 6 juillet 2015.

Kaufmann, Jean-Claude. La femme seule et le Prince charmant. Enquête sur la vie en solo. Paris : Nathan, 1999.

Kerebel, Anne. "'Claviers intimes' : les journaux en ligne comme nouvel espace d'intimité ». RiLUnE 5 (2006) : 107-120.

Kinsella, Sophie. Confessions d'une accro du shopping. Traduit de l'anglais par Isabelle Vassart. Paris : Pocket, 2004.

Labé, Yves-Marie, «"Cadavre exquis", de Pénélope Bagieu : l'hôtesse et le romancier. » Le Monde des Livres. 6 mai 2010. Web. 13 juillet 2015.

Lallemand, Caroline. «Bullez les filles !» Le VifWeekend. 1 mai 2009 : 20-24.

Lemaire, Thierry. "Margaux Motin. 'Le cœur du sujet, c'est cette nana qui part à la recherche d'elle-même'. » ActuaBD. 24 juin 2013. Web. 10 juillet 2015.

Lemoine, Clément. "Poussée de croissance "girly" ». Neuvième art 2.0. septembre 2011. Web. 13 juillet 2015.

Locatelli, Julie. «MonEspace. » Muteen 69 (2008) : 139.

Locatelli, Julie. « MonEspace. » Muteen 74 (2008) : 138.

Locatelli, Julie. «She's back. ». Muteen 80 (2009) : 26.

Martin, Madeleine. Les madeleines de Mady. Web. 6 juillet 2015.

Motin, Margaux. J'aurais adoré être ethnologue. Paris : Marabout, 2009.

Motin, Margaux. La théorie de la contorsion. Paris : Marabout, 2010.

Motin, Margaux. La tectonique des plaques. Paris : Delcourt, 2013.

Motin, Margaux. Margeaux Motin. Web. 6 juillet 2015.

Norot, Anne-Claire. "Nine Antico dynamite la BD girly .» Les inRocks. 23 octobre 2010. Web. 6 juillet 2013.

Olivier, Séverine. "La chick lit ou les mémoires d'une jeune femme “dérangée". » Belphégor 6.2 (2007). Web. 13 juillet 2015.

Olivier, Séverine. "De la presse féminine à la chick lit: un rapport de genre(s) ». In Andrin, Muriel, Brogniez Laurence et al. Femmes et critique(s). Lettres, Arts, Cinéma. Namur: Presses universitaires de Namur, 2009. 75-89.

Pasamonik, Didier. «Myriam Rak ("Mimi Stinguette”) : "Devoir passer son temps à se justifier de ne pas être une sotte superficielle n'est pas à mes yeux une avancée vers l'égalité." "ActuaBD. 7 septembre 2011. Web. 10 juillet 2015.

Pasamonik, Didier. «Bisou, le pari "girly" de Delcourt. ». ActuaBD. 5 avril 2013. Web.10 juillet 2015.

Picault, Aude. Eva. J.F. se cherche désespérément. Paris : Glénat, 2008.

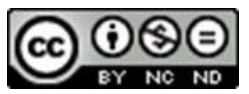


Pudlowski, Charlotte. «Faut-il en finir avec la BD girly ?». 13 octobre 2011. Web. 14 octobre 2011. Web. 5 juin 2015

Reber, Allison. «Aude Picault, jeune femme à la mer ». Bodoï. 29 mai 2009. Web. 8 juillet 2015.

Rollin, Eva. Mademoiselle. 1. Manuel de la célibataire imparfaite. Québec : Glénat, 2008.

Rollin, Eva. Mademoiselle. 4. L'arme fotale. Québec : Glénat, 2011.

Rollin, Eva. Eva Rollin. Web. 6 juillet 2015.

Schmidt, Florence. «La croqueuse de nanas.» Femina. 14 septembre 2009 : 18-19.

Tilleul, Jean-Louis. "Auteures de bande dessinée: les ambiguïtés persistantes du discours critique ». In Andrin, Muriel, Brogniez, Laurence et al. Femmes et critique(s). Lettres, Arts, Cinéma. Namur : Presses universitaires de Namur, 2009. 117-142.

Rak, Myriam. Mimi Stinguette. Web. 6 juillet 2015.

Rouquette, Sébastien. «Les blogues BD, entre blogue et bande dessinée. ». Hermès, La Revue 2.54 (2009) : 119-124. Web. 23 mai 2015.

Voflson, Olga. «BD : La dessinatrice Diglee nous présente "Forever Bitch", son dernier album. » Grazia. 29 octobre 2013. Web. 6 juillet 2015. 